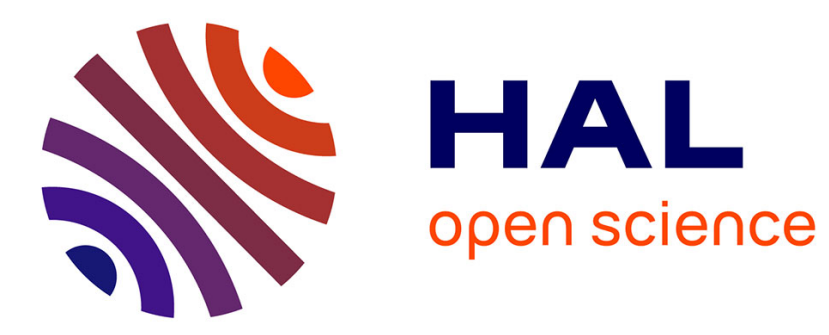

\title{
Aspects théorique et pratique des échanges thermiques dans la cavité vitre-absorbeur d'un capteur plan
}

\author{
A. Alexandre, J. Martinet
}

\section{To cite this version:}

A. Alexandre, J. Martinet. Aspects théorique et pratique des échanges thermiques dans la cavité vitre-absorbeur d'un capteur plan. Revue de Physique Appliquée, 1979, 14 (1), pp.81-85. 10.1051/rphysap:0197900140108100 . jpa-00244593

\section{HAL Id: jpa-00244593 https://hal.science/jpa-00244593}

Submitted on 1 Jan 1979

HAL is a multi-disciplinary open access archive for the deposit and dissemination of scientific research documents, whether they are published or not. The documents may come from teaching and research institutions in France or abroad, or from public or private research centers.
L'archive ouverte pluridisciplinaire HAL, est destinée au dépôt et à la diffusion de documents scientifiques de niveau recherche, publiés ou non, émanant des établissements d'enseignement et de recherche français ou étrangers, des laboratoires publics ou privés. 


\title{
Aspects théorique et pratique des échanges thermiques dans la cavité vitre-absorbeur d'un capteur plan
}

\author{
A. Alexandre et J. Martinet \\ Université de Poitiers (UER ENSMA), Laboratoire d'Energétique Solaire, 86034 Poitiers, France
}

(Reçu le 2 mai 1978, révisé le 12 octobre 1978, accepté le 16 octobre 1978)

\begin{abstract}
Résumé. - Le but de cet exposé est de montrer comment la méthode d'échanges radiatifs de Poljak au sein d'une cavité peut être étendue au cas de capteurs solaires plans à effet de serre [1], et ce dans le but de mettre en évidence les échanges radiatifs internes permettant un calcul aisé du rendement de capteur, rendement que l'on compare à celui mesuré par voie expérimentale.
\end{abstract}

\begin{abstract}
The purpose of this paper is to show how the method of Poljak's radiation exchanges in a cavity can be applied to flat solar collectors in order to render evident the internal radiative exchanges and to allow an easy calculation of collector's efficiency. This can be compare with the experimental efficiency.
\end{abstract}

1. Généralités. Aspect théorique. - Les calculs classiques relatifs au bilan thermique dans une enceinte opaque font habituellement appel aux hypothèses suivantes à savoir que chaque surface ou élément de surface est isotherme, diffusément gris et de plus uniformément irradié, ce qui implique une certaine discrétisation des surfaces radiatives, la dernière condition étant souvent plus contraignante que la seconde.

Encore que l'assimilation d'un capteur vitré à effet de serre à une cavité opaque ne soit rigoureusement valable que pour les seules radiations infrarouges (de longueur d'onde supérieure à $3 \mu\left({ }^{1}\right)$ ) pour lesquelles le vitrage a un comportement opaque, cela implique aussi la particularisation des éléments soumis à l'irradiation solaire directe et la possibilité de séparer nettement les deux types de radiations solaires et infrarouges (ces dernières correspondant à des émissions de surfaces dont les températures ne dépassent pas $400 \mathrm{~K}\left({ }^{2}\right)$ ). Il est donc normal de tenir compte de la variation des facteurs d'émission et de réflexion en fonction du rayonnement pris en considération et de songer à appliquer l'une des trois méthodes classiques

( ${ }^{1}$ ) Le verre usuel présente un créneau de transparence pour les radiations de longueur d'onde $0,3-3 \mu$, son comportement est opaque au-delà.

(2) Cette séparation se justifie par les faits que :

$93,9 \%$ de l'énergie solaire d'irradiation correspond à des radiations de longueur d'onde $<2 \mu$,

$99,5 \%$ de l'énergie solaire d'irradiation correspond à des radiations de longueur d'onde $<5 \mu$,

et que la température des différentes surfaces de l'enceinte ne dépassant pas $400 \mathrm{~K}$, seulement $5 \times 10^{-3} \%$ de l'énergie d'émission correspond à des longueurs d'onde $>2 \mu$. de Poljak, Gebhart ou Hottel, la première ayant toutefois l'avantage de présenter un sens physique plus immédiat, du fait de l'utilisation des radiosités adimensionnelles correspondant à des états d'équilibres partiels [2].

Chaque élément de surface devra alors toutefois présenter en plus des conditions déjà évoquées relativement à l'isothermicité et l'uniformité de l'irradiation, un caractère doublement gris et diffus, le premier relativement aux radiations infrarouges, le second relativement aux radiations solaires dès leur première réflexion.

Ceci étant, si le repérage des éléments de surface s'effectue au moyen de l'indice $i(l \leqslant i \leqslant N)$ et si l'on désigne par :

$T_{i} \quad$ la température absolue de l'élément $i$,

$\rho_{i}^{\mathrm{p}}=1-\varepsilon_{i}^{\mathrm{p}}$ son facteur de réflexion propre,

$\rho_{i}^{s}$ son facteur de réflexion relativement aux radiations solaires,

$e_{i}^{s}$ la densité de flux solaire irradiant directement la surface de l'élément $i$,

$q_{i}$ le bilan radiatif (compté positivement s'il s'agit d'un apport) relatif à la surface unitaire de cet élément, c'est-à-dire l'écart entre l'énergie absorbée et l'énergie propre émise,

$F_{i j} \quad$ le facteur de forme relatif aux éléments $i$ et $j$,

$B_{i}^{p}$ la radiosité propre, c'est-à-dire ayant son origine dans les seules émissions propres au capteur et ainsi liée aux températures des différents éléments,

$B_{i}^{\mathbf{s}} \quad$ la radiosité solaire, c'est-à-dire ayant son origine dans le seul rayonnement solaire indépendamment du champ thermique interne au capteur,

$B_{i}$ la radiosité totale, c'est-à-dire le flux total quittant l'élément $i\left(B_{i}=B_{i}^{\mathrm{s}}+B_{i}^{\mathrm{p}}\right)$. 
La formulation générale des radiosités et bilans s'exprime comme suit, relativement à l'unité de surface de l'élément $i$, et ce, compte tenu d'une part de la séparation des rayonnements et d'autre part des relations de réciprocité entre facteurs de forme :

$$
\begin{aligned}
B_{i}= & B_{i}^{\mathrm{p}}+B_{i}^{\mathrm{s}}=\sigma \varepsilon_{i}^{\mathrm{p}} T_{i}^{4}+\rho_{i}^{\mathrm{p}} \sum_{j=i}^{N} B_{j}^{\mathrm{p}} F_{i j}+ \\
& +\rho_{i}^{\mathrm{s}} \sum_{j=1}^{N} B_{j}^{\mathrm{s}} F_{i j} \\
q_{i}= & \left(1-\rho_{i}^{\mathrm{p}}\right) \sum_{j=1}^{N} B_{j}^{\mathrm{p}} F_{i j}+ \\
& +\left(1-\rho_{i}^{\mathrm{s}}\right) \sum_{j=1}^{N} B_{j}^{\mathrm{s}} F_{i j}-\sigma \varepsilon_{i}^{\mathrm{p}} T_{i}^{4} .
\end{aligned}
$$

Ceci étant, introduisons les radiosités adimensionnelles :

$\beta_{j}^{\mathrm{p}(i)} \quad$ correspondant au quotient par $\sigma T_{i}^{4}$ de la radiosité propre quittant l'élément $j$ lorsque les températures de tous les éléments autres que $i$ sont nulles.

$\beta_{j}^{\mathrm{s}(i)} \quad$ correspondant au quotient par $\sigma T_{i}^{4}$ de la radiosité solaire quittant l'élément $j$ lorsque les températures de tous les éléments sont nulles et que seule la surface $i$ est irradiée.

Dans ces conditions, la radiosité totale relative à la surface $i$ et correspondant à la superposition des états d'équilibre partiels est donnée par :

$B_{i}=\sum_{k=1}^{N}\left[\beta_{i}^{\mathrm{p}(k)}+\beta_{i}^{\mathrm{s}(k)}\right] \sigma T_{k}^{4}$

La connaissance des $2 n^{2}$ termes de radiosité adimensionnelle est obtenue par la résolution des systèmes linéaires suivants :

$$
\begin{aligned}
& 1 \leqslant j \leqslant N\left\{\beta_{i \neq j}^{\mathrm{p}(j)}=\rho_{i}^{\mathrm{p}} \sum_{k=1}^{N} \beta_{k}^{\mathrm{p}(j)} F_{i k}\right. \\
& 1 \leqslant i \leqslant N\left\{\beta_{j}^{\mathrm{p}(j)}=\varepsilon_{j}^{\mathrm{p}}+\rho_{j}^{\mathrm{p}} \sum_{k=1}^{N} \beta_{k}^{\mathrm{p}(j)} F_{j k}\right. \\
& \begin{array}{l}
1 \leqslant j \leqslant N \\
1 \leqslant i \leqslant N
\end{array}\left\{\begin{array}{l}
\beta_{i \neq j}^{\mathrm{s}(j)}=\rho_{i}^{\mathrm{s}} \sum_{k=1}^{N} \beta_{k}^{\mathrm{s}(j)} F_{i k} \\
\beta_{j}^{\mathrm{s}(j)}=\rho_{j}^{\mathrm{s}} \sum_{k=1}^{N} \beta_{k}^{\mathrm{s}(j)} F_{j k}+\frac{\rho_{j}^{\mathrm{s}} e_{j}^{\mathrm{s}}}{\sigma T_{j}^{4}} .
\end{array}\right.
\end{aligned}
$$

En reportant ces valeurs dans les équations (2) et (3) tout en y incluant les principes de conservation de l'énergie interne et des échanges radiatifs équivalents entre deux surfaces portées à la même température, nous obtenons :

$$
\begin{aligned}
q_{i}= & \frac{\varepsilon_{i}^{\mathrm{p}}}{1-\varepsilon_{i}^{\mathrm{p}}} \sum_{k=1}^{N} \beta_{i}^{\mathrm{p}(k)} \sigma\left(T_{i}^{4}-T_{k}^{4}\right)- \\
& -\frac{1-\rho_{i}^{\mathrm{s}}}{\rho_{i}^{\mathrm{s}}} \sum_{k=1}^{N} \beta_{i}^{\mathrm{s}(k)} \sigma T_{k}^{4} .
\end{aligned}
$$

2. Aspect pratique. - Le type de capteur choisi est essentiellement constitué par des tubes de polychlorure de vinyle (PVC) parcourus par une circulation d'eau et reposant sur une tôle ondulée en fer galvanisé, elle-même reposant sur une couche de polystyrène expansé, la partie supérieure de l'ensemble étant coiffée par une vitre - les dimensions et l'aspect ressortent figure 1 et sur les photos $\mathrm{A}, \mathrm{B}$ et $\mathrm{C}$. Le développement de ce capteur (amené jusqu'à sa phase industrielle) a été conditionné par les réalités économiques des pays du tiers monde et plus précisément à Madagascar.

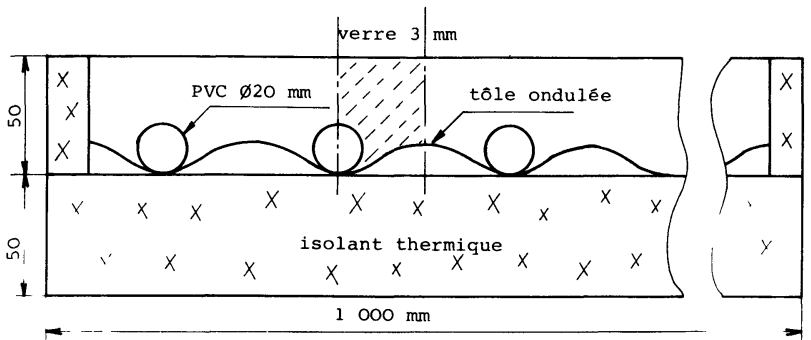

Fig. 1. - Coupe transversale du capteur expérimental.

[Transversal section of experimental collector.]

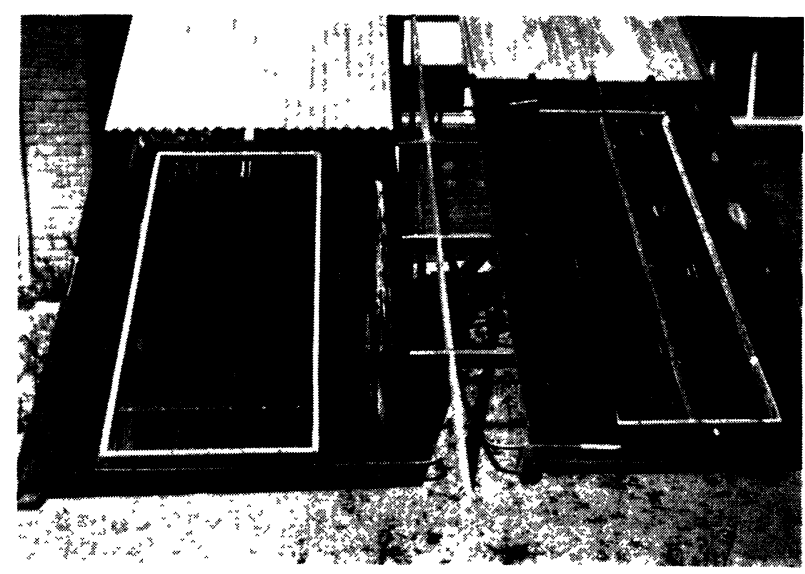

Photo A. - Vue générale d'un capteur expérimental.

[General view of experimental collector.]

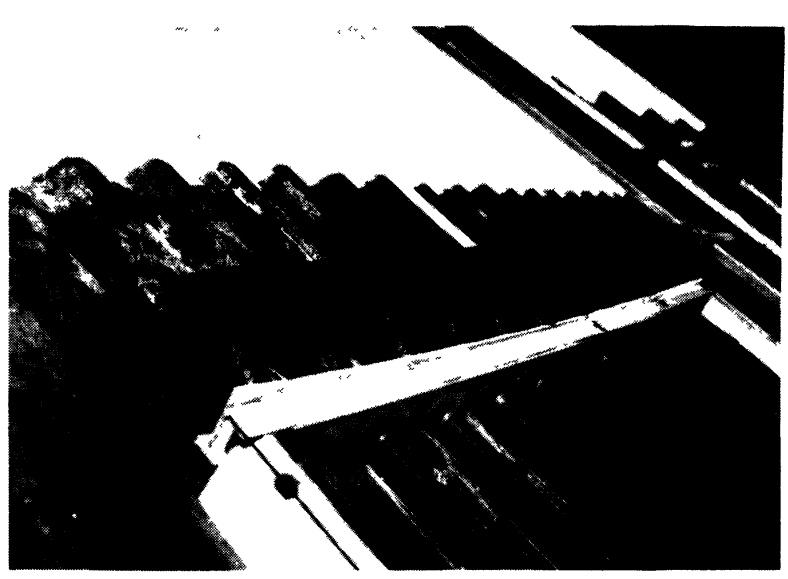

Photo B. - Détail du collecteur supérieur.

[Detail of upper pipe.] 


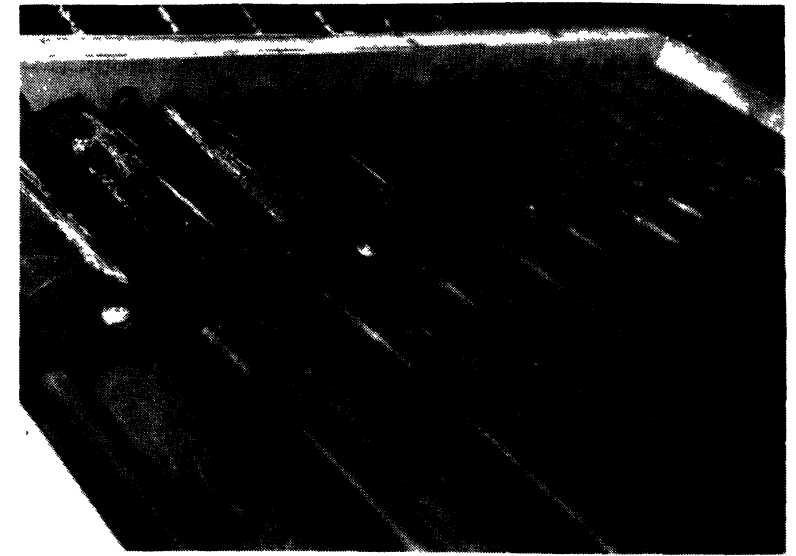

Photo C. - Détail de la fixation des canalisations.

[Details of pipes fixations.]

La modélisation du capteur s'effectue dans les conditions suivantes : les données sont essentiellement d'une part, l'énergie d'irradiation directe et la température de l'air extérieur (données externes) et d'autre part, le champ thermique stationnaire que constitue la connaissance des températures des surfaces irradiées. Celles-ci s'effectuent par voie thermoélectrique (implantation de thermocouples par collage sur le PVC et la tôle métallique, implantation par collage sur le verre après laminage des fils et protection locale de la soudure contre l'irradiation directe). Ces données permettent un calcul relativement aisé des bilans et rendements. Il n'en serait pas de même du problème où la dernière série de données (internes) serait remplacée par une donnée externe (débit et température moyenne d'entrée du fluide caloporteur dans les canalisations par exemple), la complexité de résolution s'avérant alors beaucoup plus grande.

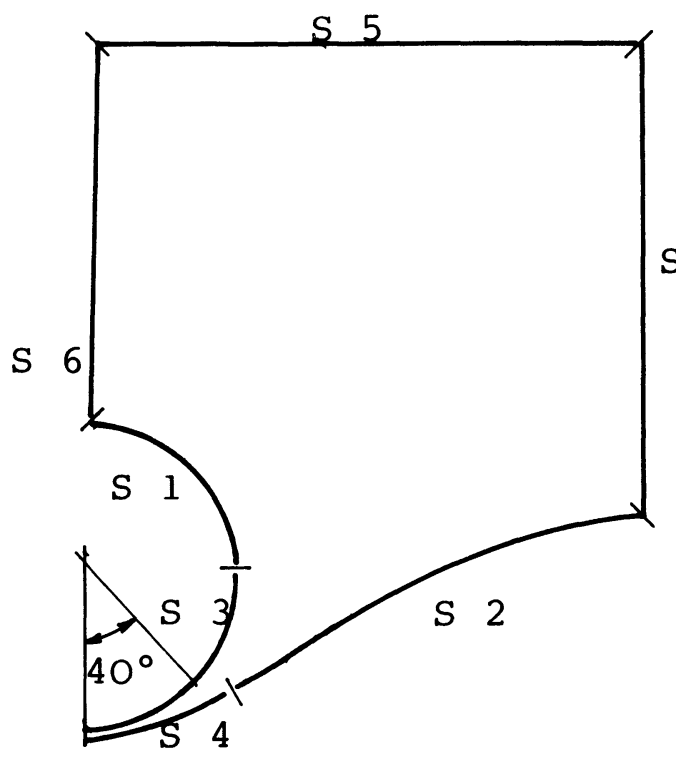

Fig. 2. - Cavité rayonnante.

[Radiative cavity.]
La figure 2 représente le schéma utilisé lors de la discrétisation des surfaces. L'influence tenue pour négligeable des effets de bord, l'isothermicité des surfaces apparaissant dans ce découpage et l'uniformité de l'insolation (toutes choses qui ont été vérifiées expérimentalement) ont permis de délimiter une cavité rayonnante de développement axial. En effet, le gradient de température longitudinal est faible aussi bien sur la tôle que sur les canalisations PVC l'écart ne dépassant pas 3 à $4{ }^{\circ} \mathrm{C}$ pour 2 points situés sur une même ligne longitudinale et espacés de $1,50 \mathrm{~m}$; transversalement les surfaces délimitées ont un écart de température extrême n'excédant pas $1^{\circ} \mathrm{C}$. Dans cette cavité interviennent les deux surfaces fictives $S_{6}$ et $S_{7}$. Du fait de la symétrie du phénomène thermique on peut attribuer à ces deux surfaces un caractère d'adiabaticité (ce qui revient à considérer ces surfaces comme parfaitement réfléchissantes, donc de facteur d'émission nul). Il en résulte pour l'équation (5) la forme suivante :

$$
q_{i}=\frac{\varepsilon_{i}^{\mathrm{p}}}{1-\varepsilon_{i}^{\mathrm{p}}} \sum_{k=1}^{5} \beta_{i}^{\mathrm{p}(k)} \sigma\left(T_{i}^{4}-T_{k}^{4}\right)-\frac{1-\rho_{i}^{\mathrm{s}}}{\rho_{i}^{\mathrm{s}}} \sum_{k=1}^{2} \beta_{i}^{\mathrm{s}(k)} \sigma T_{k}^{4}
$$

Le bilan calorifique relatif à la demi-canalisation (surfaces 1 et 3 ) n'est alors autre que la somme des deux termes $q_{1}$ et $q_{3}$ dont la connaissance nécessite celle des radiosités réduites

$$
\begin{array}{llll}
\beta_{1}^{\mathrm{p}(i)} & \beta_{3}^{\mathrm{p}(i)} & \beta_{1}^{\mathrm{s}(i)} & \beta_{3}^{\mathrm{s}(i)} \\
0<i \leqslant 5 & 0<i \leqslant 5 & i=1,2 & i=1,2 .
\end{array}
$$

Or, les deux premières valeurs s'obtiennent indépendamment de toute valeur expérimentale puisque ne faisant intervenir que des facteurs d'émission et de réflexion propre; les facteurs de formes se calculent aisément par la méthode classique de Hottel (demidifférence des longueurs des lignes croisées et non croisées) (Tableau I). Les facteurs d'émission propres ont été fixés selon les valeurs fournies par les tables à 0,88 pour le verre et à 0,95 pour la peinture noire (à ce dernier revêtement correspond le facteur de réflexion solaire $\rho^{\mathrm{s}}$, choisi dans les mêmes conditions,

\begin{tabular}{|c|c|c|c|c|c|c|c|}
\hline$F_{i j \rightarrow}$ & 1 & 2 & 3 & 4 & 5 & 6 & 7 \\
\hline - & - & - & - & - & - & - & - \\
\hline 1 & 0 & 0,190 & 0 & 0 & 0,268 & 0,388 & 0,153 \\
\hline 2 & 0,09 & 0 & 0,149 & 0,006 & 0,301 & 0,206 & 0,248 \\
\hline 3 & 0 & 0,313 & 0 & 0,589 & 0,013 & 0,085 & 0 \\
\hline 4 & 0 & 0,019 & 0,850 & 0 & 0,029 & 0,062 & 0 \\
\hline 5 & 0,106 & 0,249 & 0,005 & 0,008 & 0 & 0,325 & 0,308 \\
\hline 6 & 0,122 & 0,136 & 0,027 & 0,013 & 0,260 & 0 & 0,442 \\
\hline 7 & 0,053 & 0,182 & 0 & 0 & 0,273 & 0,491 & 0 \\
\hline
\end{tabular}
égal à 0,15 ). La peinture noire courante utilisée a été

Tableau I. - Valeur des facteurs de forme dans le capteur. 
exposée longuement aux radiations solaires sous vitrage afin de se rapprocher au maximum des conditions usuelles d'utilisation. Il est à noter que des tests de calculs montrent une faible sensibilité paramétrique (moins de $10 \%$ de variation de puissance pour $10 \% \mathrm{de}$ variation du facteur de réflexion). Quant aux échanges de moindre importance (conductifs et convectifs) on les détermine d'une manière approchée en admettant que la malléabilité des matériaux utilisés n'autorise pas un véritable contact thermique entre la canalisation et son support métallique, ce qui implique :

- pour les échanges conductifs de s'opérer sur un arc de cercle de $80^{\circ}$ environ traduisant la similitude des profils comme nous le montre la figure 1, à travers une fine lame d'air immobile d'épaisseur moyenne $0,5 \mathrm{~mm}$ et de conductivité thermique $\lambda a$,

- pour les échanges convectifs de pouvoir se chiffrer à partir de l'expression suivante donnant le coefficient de transfert thermique local d'échange externe :

$h_{\mathrm{c}}(\alpha)=\frac{\lambda a}{D} \cdot 0,604 G r_{D}^{0,25} \varphi(\alpha)$

où $D$ est le diamètre du cylindre,

$G r_{D}$ est le nombre de Grashof relatif au diamètre $D$.

$\varphi(\alpha)$ est une fonction [3] dépendant de l'angle $\alpha$ et dont la valeur varie de $0,75\left(\alpha=-60^{\circ}\right)$ à $0,66(\alpha=0)$ et $0,46\left(\alpha=+60^{\circ}\right)$.

Les résultats relatifs aux surfaces 1 et 3 d'un capteur de $1 \mathrm{~m}^{2}$ de surface portant sur les différents apports radiatifs, conductifs et convectifs ont été, pour différentes valeurs de l'énergie d'irradiation, groupés sur le tableau II. Sur ce même tableau figurent les rendements théoriques obtenus par voie de calcul $(\S 1)$, et par voie expérimentale par mesure de l'élévation de température du fluide caloporteur et de son débit.

Ce tableau mentionne également l'écart de température entre l'air extérieur et le fluide caloporteur, écart dont dépend le rendement. En effet, expérimentalement on constate que les courbes de rendement paramétrées par le flux solaire incident, en fonction de

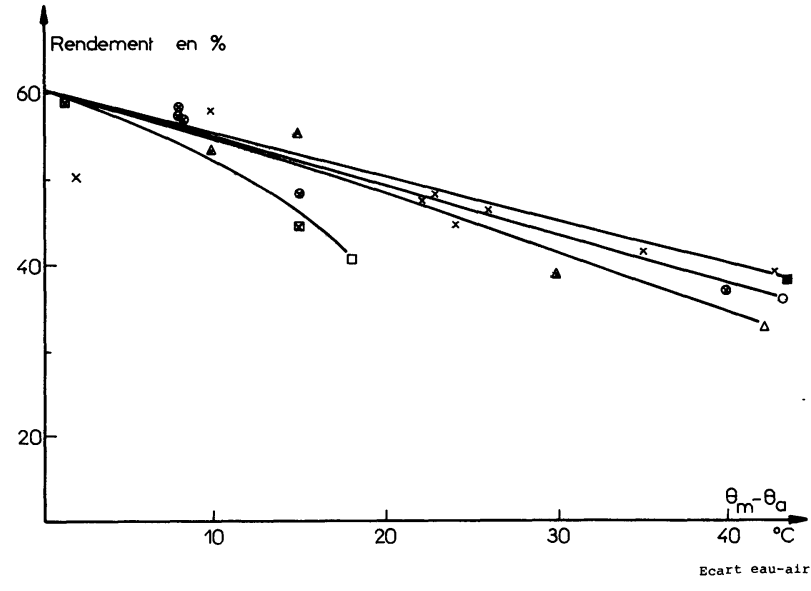

Fig. 3. - Résultats théoriques et expérimentaux concernant le rendement du capteur solaire. - Courbe expérimentale $Q_{\mathrm{s}}=925-1040 \mathrm{~W} / \mathrm{m}^{2}$. Points obtenus par calcul $\times$. $\circ$ Courbe expérimentale $Q_{\mathrm{s}}=810-925 \mathrm{~W} / \mathrm{m}^{2}$. Points obtenus par calcul $\otimes$. $\Delta$ Courbe expérimentale $Q_{\mathrm{s}}=695-810 \mathrm{~W} / \mathrm{m}^{2}$. Points obtenus par calcul $\Delta . \square$ Courbe expérimentale $Q_{\mathrm{s}}=580-695 \mathrm{~W} / \mathrm{m}^{2}$. Points obtenus par calcul $\bigotimes$

[Experimental and theoretical results relating to the solar collectors efficiency.]

Tableau II. - Répartition des flux de rayonnement, de conduction et de convection sur les canalisations du capteur.

[Division of radiation conduction and convection flux on canalization of solar collector.]

\begin{tabular}{|c|c|c|c|c|c|c|c|c|}
\hline $\begin{array}{l}\text { Puissance solaire } \\
\text { d'irradiation } \\
\mathrm{W} \mathrm{m}^{-2}\end{array}$ & $\begin{array}{l}\text { Puissance rayon- } \\
\text { nante absorbée } \\
\text { par les demi- } \\
\text { canalisations } \\
\text { supérieures } \\
\left(\mathrm{W} \mathrm{m} \mathrm{m}^{-2}\right)\end{array}$ & $\begin{array}{c}\text { Puissance } \\
\text { rayonnante } \\
\text { absorbẹe par les } \\
\text { demi-canalisa- } \\
\text { tions inférieures } \\
\left(\mathrm{W} \mathrm{m}^{-2}\right)\end{array}$ & $\begin{array}{l}\text { Puissance } \\
\text { apportée par } \\
\text { conduction } \\
\mathrm{W} \mathrm{m}^{-2}\end{array}$ & $\begin{array}{c}\text { Puissance } \\
\text { apportée } \\
\text { par } \\
\text { convection } \\
\mathrm{W} \mathrm{m}^{-2}\end{array}$ & $\begin{array}{c}\text { Puissance } \\
\text { totale absorbée } \\
\text { par tubes } \\
\mathrm{W} \mathrm{m}^{-2}\end{array}$ & \begin{tabular}{|c|} 
Rendement \\
par voie \\
de calcul \\
en $\%$
\end{tabular} & $\begin{array}{c}\text { Rendement } \\
\text { obtenu } \\
\text { expérimen- } \\
\text { talement } \\
\text { en } \%\end{array}$ & $\begin{array}{c}\text { Ecart de } \\
\text { température } \\
\text { eau-air } \\
\text { en }{ }^{\circ} \mathrm{C}\end{array}$ \\
\hline- & - & - & - & - & - & - & - & - \\
\hline 1010 & 204 & 71 & 176 & 34 & 485 & 48 & 48 & 23 \\
\hline 1010 & 200 & 60 & 150 & 29 & 439 & 44 & 47 & 24 \\
\hline 1000 & 195 & 68 & 176 & 34 & 473 & 47 & 49 & 22 \\
\hline 998 & 187 & 56 & 142 & 27 & 412 & 41 & 41 & 35 \\
\hline 998 & 199 & 67 & 167 & 31 & 464 & 46 & 46 & 26 \\
\hline 995 & 214 & 64 & 186 & 35 & 499 & 50 & 58 & 2 \\
\hline 975 & 205 & 56 & 132 & 30 & 423 & 44 & 51 & 15 \\
\hline 949 & 217 & 87 & 215 & 35 & 554 & 58 & 54 & 10 \\
\hline 929 & 180 & 53 & 187 & 28 & 448 & 48 & 51 & 15 \\
\hline 894 & 207 & 88 & 183 & 37 & 515 & 58 & 55 & 8 \\
\hline 870 & 202 & 92 & 180 & 35 & 509 & 58 & 55 & 8 \\
\hline 870 & 202 & 92 & 180 & 35 & 509 & 58 & 55 & 8 \\
\hline 786 & 176 & 67 & 171 & 19 & 433 & 55 & 51 & 15 \\
\hline 722 & 146 & 30 & 167 & 35 & 378 & 53 & 54 & 10 \\
\hline 376 & 85 & 30 & 87 & 21 & 223 & 59 & 59 & 1,5 \\
\hline
\end{tabular}

Les calculs des colonnes 2 à 7 ont été effectués à partir d'une irradiation réelle consignée, colonne 1, entraînant un champ thermique, mesuré, parfaitement défini dans le capteur. 
l'écart eau-air, sont reproductibles pour des variations du niveau de température de $\pm 5^{\circ} \mathrm{C}$, ce qui fut le cas lors de nos expériences (Fig. 3).

3. Conclusion. - Le schéma développé ci-dessus applicable à tout capteur, quelle que soit sa géométrie, a pour principal mérite sa simplicité et l'accord relativement bon entre le calcul et les résultats expérimentaux (les écarts ne dépassent guère $10 \%$ dans les cas les plus défavorables). Il est à noter que cet accord a été obtenu malgré nombre d'hypothèses dont le bien-fondé semblait parfois discutable.

Par ailleurs, il est intéressant de constater que les calculs font apparaître au niveau de l'eau de circulation un apport calorifique qui, pour plus de $60 \%$, est redevable de l'échange radiatif, alors que la surface de canalisation exposée au rayonnement solaire ne représente que $26 \%$ de la surface totale (Fig. 1), mettant ainsi en évidence le rôle majeur que jouent les échanges radiatifs, et ce, même dans le cas de températures inférieures à $100^{\circ} \mathrm{C}$ et ne s'écartant guère de plus de 10 à $20^{\circ} \mathrm{C}$ les unes des autres.

\section{Bibliographie}

[1] Alexandre, A., Contribution à l'étude et au développement de capteurs plans, Thèse d'ingénieur docteur U.E.R. ENSMA, Université de Poitiers.

[2] SPARrow, Modern developments in heat transfer (Academic Press), p. 181.

[3] Kreith, F., Transmission de la chaleur et thermodynamique (Masson et Cie), p. 354. 